\title{
Gain-of-function mutation in the KCNMB1 potassium channel subunit is associated with low prevalence of diastolic hypertension
}

\author{
José M. Fernández-Fernández,1 Marta Tomás, ${ }^{2}$ Esther Vázquez, ${ }^{1}$ Patricio Orio, ${ }^{3}$ Ramón Latorre, ${ }^{3}$ \\ Mariano Sentí, ${ }^{1,2}$ Jaume Marrugat, ${ }^{2}$ and Miguel A. Valverde ${ }^{1}$ \\ 1 Unitat de Senyalització Cel.lular, Departament de Ciències Experimentals i de la Salut, Universitat Pompeu Fabra, Barcelona, Spain. \\ 2Unitat de Lípids i Epidemiologia Cardiovascular, Institut Municipal d'Investigació Mèdica, Barcelona, Spain. ${ }^{3}$ Centro de Estudios Científicos, Valdivia, Chile.
}

\begin{abstract}
Hypertension is the most prevalent risk factor for cardiovascular diseases, present in almost $30 \%$ of adults. A key element in the control of vascular tone is the large-conductance, $\mathrm{Ca}^{2+}$-dependent $\mathrm{K}^{+}(\mathrm{BK})$ channel. The $\mathrm{BK}$ channel in vascular smooth muscle is formed by an ion-conducting $\alpha$ subunit and a regulatory $\beta_{1}$ subunit, which couples local increases in intracellular $\mathrm{Ca}^{2+}$ to augmented channel activity and vascular relaxation. Our large population-based genetic epidemiological study has identified a new single-nucleotide substitution (G352A) in the $\beta_{1}$ gene (KCNMB1), corresponding to an $\mathrm{E} 65 \mathrm{~K}$ mutation in the protein. This mutation results in a gain of function of the channel and is associated with low prevalence of moderate and severe diastolic hypertension. $\mathrm{BK}-\beta_{1 \mathrm{E} 65 \mathrm{~K}}$ channels showed increased $\mathrm{Ca}^{2+}$ sensitivity, compared with wild-type channels, without changes in channel kinetics. In conclusion, the $B K-\beta_{1 \mathrm{E} 65 \mathrm{~K}}$ channel might offer a more efficient negativefeedback effect on vascular smooth muscle contractility, consistent with a protective effect of the $\mathrm{K}$ allele against the severity of diastolic hypertension.
\end{abstract}

\section{Introduction}

High blood pressure, or hypertension, is not only a disease but a risk factor for cardiac, brain, and kidney pathology as well $(1,2)$. Considering that between $25-40 \%$ of the adult population is hypertensive, and more than $90 \%$ of cases are of unknown origin, i.e., essential hypertension, the identification of molecular mechanisms involved in its pathophysiology and predictors of hypertension is of paramount relevance for the management of public health and the development of antihypertensive treatments. Despite efforts exploring genetic susceptibility to essential hypertension, the search for candidate genes with major repercussion at the population level has proven difficult and disappointing (3-5), reflecting the multifactorial nature of the hypertensive phenotype $(6,7)$.

The blood pressure within arteries depends on the resistance of the vessels, i.e., the diameter of the blood vessels, which in turn is controlled by the contraction of the smooth muscle cells in their walls (the arterial tone). The control of arterial tone depends on a calcium signal in the vascular smooth muscle, mainly provided by the influx of $\mathrm{Ca}^{2+}$ via voltage-gated channels and its release from intracellular stores (8). A key element in the control of the vascular tone is the large-conductance, $\mathrm{Ca}^{2+}$ - and voltage-dependent $\mathrm{K}^{+}(\mathrm{BK})$ channel, which couples local increases in intracellular $\mathrm{Ca}^{2+}$ to augmented channel activity and vascular relaxation $(8,9)$. At the molecular level, the BK channel in vascular smooth muscle is formed by an ion-conducting $\alpha$ subunit (10) and a regulatory $\beta_{1}$ subunit (11-13). In smooth muscle, an increase in BK channel activity is induced by local releases of $\mathrm{Ca}^{2+}$ from the sarcoplasmic reticulum ("Ca ${ }^{2+}$ sparks") that lead to hyperpolarization of the membrane and prevention of further influx of $\mathrm{Ca}^{2+}(8)$. This negative-feedback

Nonstandard abbreviations used: large conductance, $\mathrm{Ca}^{2+}$-dependent $\mathrm{K}^{+}(\mathrm{BK})$; conductance $(G)$; diastolic blood pressure (DBP); odds ratio (OR); open probability $\left(P_{\mathrm{O}}\right)$. Conflict of interest: The authors have declared that no conflict of interest exists.

Citation for this article: J. Clin. Invest. 113:1032-1039 (2004).

doi:10.1172/JCI200420347. mechanism is finely tuned by the presence of the $\beta_{1}$ subunit of the $\mathrm{BK}$ channel, which increases channel sensitivity to $\mathrm{Ca}^{2+}(11,13-17)$.

Despite the well-known negative-feedback effect of BK channels on vascular smooth muscle contraction, the only molecular evidence for its implication in the control of blood pressure comes from recent studies in animal models. Disruption of the $\beta_{1}$ gene (kcnmb1) was associated with elevated blood pressure and left ventricular hypertrophy in mouse models $(16,17)$, and downregulation of the $\beta_{1}$ subunit has been reported in hypertensive rat models $(18,19)$. However, no studies of the relationship between genetic variants of KCNMB1 and systemic blood pressure in humans have been done, although polymorphisms of KCNMB1 have been associated with baroreflex function (20). All these observations make the $K C N M B 1$ gene, coding for the $\beta_{1}$ subunit, an important target for the study of its contribution to the genetic basis of hypertension. The objective of the present study was to determine whether the KCNMB1 gene might be involved in the pathogenesis of human hypertension. For that purpose we conducted a search for genetic variants in the KCNMB1 gene together with functional ion channel studies and population-based genetic epidemiological studies.

\section{Methods}

Population study. The representative population sample was composed of 3,876 participants aged 25-74: 1,914 women (49.4\%) and 1,962 men (50.6\%). They were randomly selected in two cross-sectional studies carried out in the province of Girona, Spain, from 1994 to 1996 and from 1999 to 2001, to establish the prevalence of cardiovascular risk factors in this region. Full details have been previously provided (21). Five hundred participants were under antihypertensive drug therapy, and a further 606 had blood pressure measurements that met the WHO hypertension criteria but were unaware of their condition (483 of them with isolated diastolic hypertension). Owing to the epidemiological nature of diastolic blood pressure (DBP) measurements, which might not fit 
the clinical criteria of hypertension, only strictly normotensive subjects (DBP < $80 \mathrm{mmHg} ; n=1,727$ ) and definitely hypertensive subjects (DBP $\geq 90 \mathrm{mmHg} n=983$ ) were considered in this study. All participants gave written informed consent. The study was approved by the Institutional Ethics Committee of the Institut Municipal d'Investigació Mèdica, and the individual results were sent back to all participants.

Measured variables. A precision scale was used for weight measurement. Participants wore underwear. Height was also measured. BMI was determined as weight divided by squared height $\left(\mathrm{kg} / \mathrm{m}^{2}\right)$. The mean BMI of the participants in the study was $27.2 \mathrm{~kg} / \mathrm{m}^{2}$. Blood pressure measurements were obtained with a periodically calibrated mercury sphygmomanometer. The operator followed a certification process in the standardized measurement technique at a central laboratory, and determinations were always made by the same person. A cuff adapted to upper-arm perimeter (young, adult, obese) was selected for each participant. The first measurements were performed after a 5 -minute rest, and the second measurements were taken at least 20 minutes later. The value used was the arithmetic mean of both determinations. Standardized hypertension and diabetes mellitus questionnaires were used as described previously (21).

Screening of KCNMB1 gene. Exons encoding the modulatory $\beta_{1}$ subunit (KCNMB1) of the BK channel (22) were amplified from genomic DNA of 11 severely hypertensive and 12 strictly normotensive participants. The PCR products were analyzed by direct sequencing. The E65K variant was identified in the third exon of the KCNMB1 gene (GenBank accession no. U25138) by dideoxynucleotide-sequencing method (ABI PRISM BigDye Terminator 3.0; Applied Biosystems, Foster City, California, USA) and was confirmed by sequencing of the second strand, using the forward and reverse primers $5^{\prime}$-CAGCCAGTTAGCGGCAGATTC-3' and 5'TGTTGCAAGAGTAGCCAAGGTTG- $3^{\prime}$. Following the identification of the mutation E65K in the third exon of the KCNMB1 gene by direct sequencing, this mutation was analyzed in every one of the 3,876 participants of the study by a real-time quantitative PCR. The DNA samples were analyzed by TaqMan assay (ABI PRISM 7900HT; Applied Biosystems), using 5'-AGCGTGTGGACCCAGGAAT- $3^{\prime}$ and 5'-GGCAGCTGACACGTTGA-3' primers, and FAMCCTTCTTGCCCTTCAGCTTCTCCTC-TAMRA probe for the $\mathrm{K}$ (A base) allele and VIC-CACCTTCTTGCCCTTCAGCTCCTCTAMRA probe for the and $\mathrm{E}$ ( $\mathrm{G}$ base) allele.

Generation and expression of mutant subunits. The E65K mutation was introduced into the human $\beta_{1}$ subunit of the BK channel cloned into pcDNA3, a gift from Ligia Toro (University of California-Los Angeles, Los Angeles, California, USA), using the QuikChange Mutagenesis Kit (Stratagene, La Jolla, California, USA), and verified by subsequent sequencing. HEK-293 cells permanently expressing the human $\alpha$ subunit of the BK channel (hSlo) (23) were transfected with the BK- $\beta_{1 \mathrm{E} 65 \mathrm{~K}}$ channel construct using a linear polyethylenimine (PEI) derivative, the polycation ExGen 500 (Fermentas Inc., Hanover, Maryland, USA) (24), following the manufacturer's instructions (seven equivalents PEI per $3.3 \mu \mathrm{g}$ DNA). Two hundred fifty thousand HEK-293 cells were seeded per $35-\mathrm{mm}$ dish 24 hours before transfection. For each dish, $3 \mu \mathrm{g}$ of the $\beta_{1 \mathrm{WT}}$ or $\beta_{1 \mathrm{E} 65 \mathrm{~K}}$ construct, together with the transfection reporter coding for enhanced GFP (pEGFPN1) at a 10:1 ratio, was used. For double transfections, $1.5 \mu \mathrm{g}$ of $\beta_{1 \mathrm{wT}}$ plus $1.5 \mu \mathrm{g}$ of $\beta_{1 \mathrm{E} 65 \mathrm{~K}}$ was used.

Electrophysiology. Experiments were carried out on EGFP-positive cells plated on $35-\mathrm{mm}$ plastic dishes mounted on the stage of an Olympus IX70 inverted microscope (Olympus America Inc.,
Melville, New York, USA). Ionic currents were recorded in the inside-out patch-clamp model (25). Borosilicate glass patch pipettes had 1-3 M $\Omega$ resistance and were filled with a solution containing (in mM): $140 \mathrm{KCl}, 1.2 \mathrm{MgCl}_{2}, 0.2 \mathrm{CaCl}_{2}, 0.5 \mathrm{EDTA}$, and 10 HEPES (300 milliosmoles per liter, $\mathrm{pH}$ 7.3). Solutions bathing the cytoplasmic face of the patch membrane contained (in $\mathrm{mM}$ ): 140 $\mathrm{KCl}, 0.7 \mathrm{MgCl}_{2}$, 10 HEPES, (300 milliosmoles per liter, $\mathrm{pH} 7.25$ ). The intracellular free $\mathrm{Ca}^{2+}$ concentration (calculated using EqCal; Biosoft, Cambridge, United Kingdom) was adjusted to the desired values by different combinations of $\mathrm{CaCl}_{2}$ and EDTA added to the bath solution. Currents were acquired at $10 \mathrm{kHz}$ and low-pass-filtered at $1 \mathrm{kHz}$. For current-activation studies, membrane patches were clamped at $0 \mathrm{mV}$, pulsed for 150 milliseconds from $-100 \mathrm{mV}$ to $+200 \mathrm{mV}$ in $10-\mathrm{mV}$ steps, and repolarized to $-80 \mathrm{mV}$ for $10-20$ milliseconds. For current-deactivation studies, tail currents were evoked by a 50 -millisecond step to $+160 \mathrm{mV}$ (for $10 \mu \mathrm{M}$ cytosolic free $\mathrm{Ca}^{2+}$ ) or $+200 \mathrm{mV}$ (for $\mathrm{Ca}^{2+} \leq 1.6 \mu \mathrm{M}$ ), and then measured by repolarizing to various voltages (from $-100 \mathrm{mV}$ to $+10 \mathrm{mV}$ in 10 $\mathrm{mV}$ steps) for 50-90 milliseconds. Experiments were performed at room temperature $\left(22-26^{\circ} \mathrm{C}\right)$.

Allosteric model fitting. Assuming that the movement of the BK channel voltage sensors and $\mathrm{Ca}^{2+}$ binding occur separately from channel opening, the channel-gating kinetics is best explained by a 50-state two-tiered allosteric kinetic model, which is raised to 70 states if some coupling between $\mathrm{Ca}^{2+}$ binding and voltage-sensor movement in the same subunit is assumed (26). Despite the apparent complexity of these many states, the properties of the model in steady state can be described with only eight parameters. These are equilibrium constants for voltage-sensor activation $\left(J_{0}\right)$, channel opening $\left(L_{0}\right)$, and calcium binding $\left(K_{\mathrm{d}}\right)$; voltage-dependence factors for channel opening $\left(z_{L}\right)$ and voltage-sensor activation $\left(z_{J}\right)$; and allosteric factors for the interactions of calcium binding with channel opening $(C)$, voltage-sensor activation with channel opening $(D)$, and calcium binding with voltage-sensor activation $(E)$.

The steady-state open probability $\left(P_{\mathrm{O}}\right)$ of the channel, or the normalized conductance $(G)$ of the BK channel currents (as shown in Figure 6), at any calcium concentration and membrane potential is described by the equation

\section{Equation 1}

$$
\begin{aligned}
P_{O}(G) & =\frac{L(1+J D+K C+J K C D E)^{4}}{L(1+J D+K C+J K C D E)^{4}+(1+J+K+J K E)^{4}} \\
& =\frac{1}{1+\frac{(1+J+K+J K E)^{4}}{L(1+J D+K C+J K C D E)^{4}}}
\end{aligned}
$$

where $L=L_{0} \exp \left[\left(z_{L} \times V\right) /(k T)\right], J=J_{0} \exp \left[\left(z_{J} \times V\right) /(k T)\right]$ and $K=\left[\mathrm{Ca}^{2+}\right] / K_{\mathrm{d}} . V$ is membrane voltage, $T$ is absolute temperature, and $k=R / F(R$, Gas constant; $F$, Faraday's constant).

A satisfactory estimation of the model parameters requires more than just fitting the $P_{\mathrm{O}}(G)-V$ relationships to the equations above. Precise measurements of gating currents and open probabilities below $10^{-3}$ were used previously (26) to independently estimate $J_{0}, z_{J}, L_{0}, z_{L}$, $K_{\mathrm{d}}$, and $C$. A fit of the $P_{\mathrm{O}}(G)-V$ relationships for $\alpha+\beta_{1 \mathrm{WT}}$ channels to the model has also been published (27), but in this study, only a 50state model was used and an independent estimation of parameters is missing. Despite these limitations, the fit is reasonable, and so we attempted to fit our steady-state data to the 70-state allosteric model. 
Statistical analysis. Deviation from Hardy-Weinberg equilibrium was assessed using a $\chi^{2}$ test with 1 degree of freedom. A $\chi^{2}$ or Fisher's exact test, as appropriate, was used to compare genotype frequencies between the diastolic normotensive and hypertensive groups. Age- and sex-adjusted odds ratios (ORs) of different degrees of hypertension and their $95 \%$ confidence intervals were estimated for K-carriers (KK + KE genotypes) versus EE genotype by unconditional logistic regression analysis. $P$ values less than 0.05 were considered statistically significant. Population-attributable risk (PAR) was calculated by case-control approach, given the crosssectional nature of our study, for which the estimation of OR is similar to that obtained by the approach using incidence rates in populations (28):

\section{Equation 2}

$$
P A R \%=\frac{P_{e}(\text { OR-1 })}{1+P_{e}(\text { OR-1 })} \times 100
$$

where $P_{e}$ is the proportion of K-carrier nonhypertensive participants and OR is the age- and sex-adjusted odds ratio of diastolic hypertension for K-carriers.

Electrophysiological data are presented in graphs as mean \pm SEM. ANOVA analysis with repeated measurements was used to test differences in the voltage necessary to half activate the channel $\left(V_{1 / 2}\right)$ among cells expressing different combinations of $\alpha$ and $\beta_{1}$ subunits.

\section{Results}

Population-based genetic epidemiological study of the human KCNMB1 gene. The four exons of KCNMB1 were amplified using PCR and analyzed by direct sequencing. A new single-nucleotide substitution (G352A) corresponding to a glutamic acid-to-lysine mutation at position $65(\mathrm{E} 65 \mathrm{~K})$ of the protein was found in the third exon of the KCNMB1 gene. The genotype frequencies were $78.4 \%$ for $\mathrm{EE}$ homozygote, $20.0 \%$ for EK heterozygote, and 1.6\% for KK homozygote subjects in a representative random population sample of 3,876 participants. The observed genotype frequencies fitted the Hardy-Weinberg equilibrium. KK homozygote and EK heterozygote subjects were analyzed together because of the low prevalence of the former. Statistical analyses were performed separately for diastolic and systolic blood pressure values. Subjects with DBP lower than $80 \mathrm{mmHg}$ who were not receiving antihypertensive therapy constituted the diastolic normotensive group. The genotype frequency of the E65K mutation $(\mathrm{KK}+\mathrm{KE})$ decreased with increasing DBP values (Figure 1A), from $21.6 \%$ in the normotensive group to $3.2 \%$ in the severe hypertension group ( $\mathrm{DBP} \geq 110 \mathrm{mmHg}$ ).

The age- and sex-adjusted ORs of five levels of diastolic hypertension were estimated for the $\mathrm{K}$ allelic variant compared with the EE genotype (Figure 1B). The magnitude and the direction of the associations (OR < 1 implies a lower risk and OR > 1 implies an increased risk of hypertension) were consistent with a protective effect of the $\mathrm{K}$ allele against the severity of diastolic hypertension and with a progressively deleterious effect of the EE genotype. Indeed, there was a decreasing risk for the $\mathrm{E} 65 \mathrm{~K}$ variant with diastolic hypertension severity, which suggests a gene-dosage effect (Figure 1B). Further adjustment for diabetic status and BMI had a negligible effect on the OR values (results not shown). The population-attributable risk for the $\mathrm{K}$ allele (i.e., the reduction in diastolic hypertension prevalence that could be expected from eliminating the effect of EE genotype in the entire population) was
$-10.6 \%$ for moderate hypertension (DBP $\geq 100 \mathrm{mmHg}$ ) and $-31.8 \%$ for severe hypertension (DBP $\geq 110 \mathrm{mmHg}$ ). Similar logistic regression analysis applied to variations in systolic blood pressure showed no relationship between the E65K variant and systolic hypertension (results not shown).

Increased $\mathrm{Ca}^{2+}$ sensitivity of the BK- $\beta_{1 E 65 \mathrm{~K}}$ channels. To evaluate the mechanism by which the E65K mutation might modify BK channel activity and, therefore, its potential contribution to the control of blood pressure, we expressed and functionally tested the E65K mutant in HEK-293 cells, i.e., we measured the ionic currents generated by the movement of $\mathrm{K}^{+}$via the wild-type and mutant $\mathrm{BK}$ channels. As the frequency of the KK genotype was very low, we also evaluated the functional significance of the expression of $\beta_{1 \mathrm{E} 65 \mathrm{~K}}$ in combination with wild-type $\beta_{1}$. HEK-293 cells permanently expressing the human pore-forming $\alpha$ subunit of the BK channel (also known as hSlo1; refs. 10, 29) were transiently transfected with wildtype $\left(\beta_{1 \mathrm{WT}}\right)$, mutant $\left(\beta_{1 \mathrm{E} 65 \mathrm{~K}}\right)$, or a combination of both $\beta_{1}$ subunits $\left(\beta_{1 \mathrm{WT}}+\beta_{1 \mathrm{E} 65 \mathrm{~K}}\right)$. Figure 2 shows BK channel currents obtained from inside-out macropatches excised from transfected HEK-293 cells. The representative currents shown in Figure 2 were recorded at three different intracellular (bathing) $\mathrm{Ca}^{2+}$ concentrations: $0.1 \mu \mathrm{M}$, $1.6 \mu \mathrm{M}$, and $10 \mu \mathrm{M}$. As described previously $(11,15,30), \mathrm{BK} \alpha+\beta_{1 \mathrm{WT}}$ channels (Figure $2 \mathrm{~B}$ ) showed higher activity than channels lacking the regulatory $\beta_{1 \mathrm{WT}}$ subunit (Figure $2 \mathrm{~A}$ ). Moreover, expression of
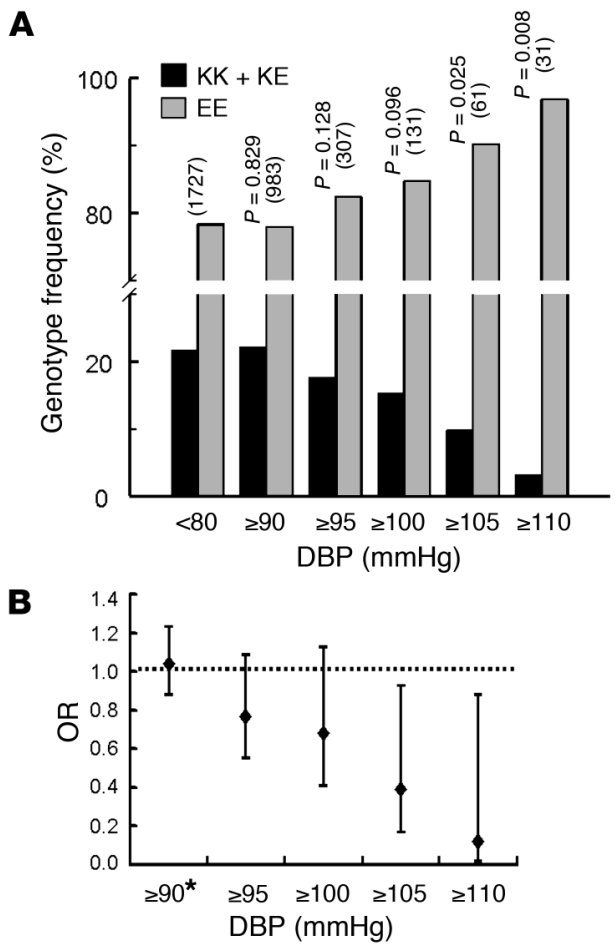

\section{Figure 1}

(A) E65K genotype distribution by DBP. $P$ values for genotype distributions at each level of diastolic hypertension are shown (with the number of subjects in parentheses). (B) Age- and sex-adjusted ORs at each level of diastolic hypertension are presented for K-carriers versus EE genotype. The adjusted ORs for K-carriers of DBP $\geq 105 \mathrm{mmHg}$ and DBP $\geq 110 \mathrm{mmHg}$ were 0.39 (95\% confidence interval, 0.17-0.93, $P=0.034$ ) and 0.12 (95\% confidence interval, 0.02-0.90, $P=0.039$ ), respectively. *Subjects under hypertension treatment with DBP $<90$ $\mathrm{mmHg}$ were also included in this group. 


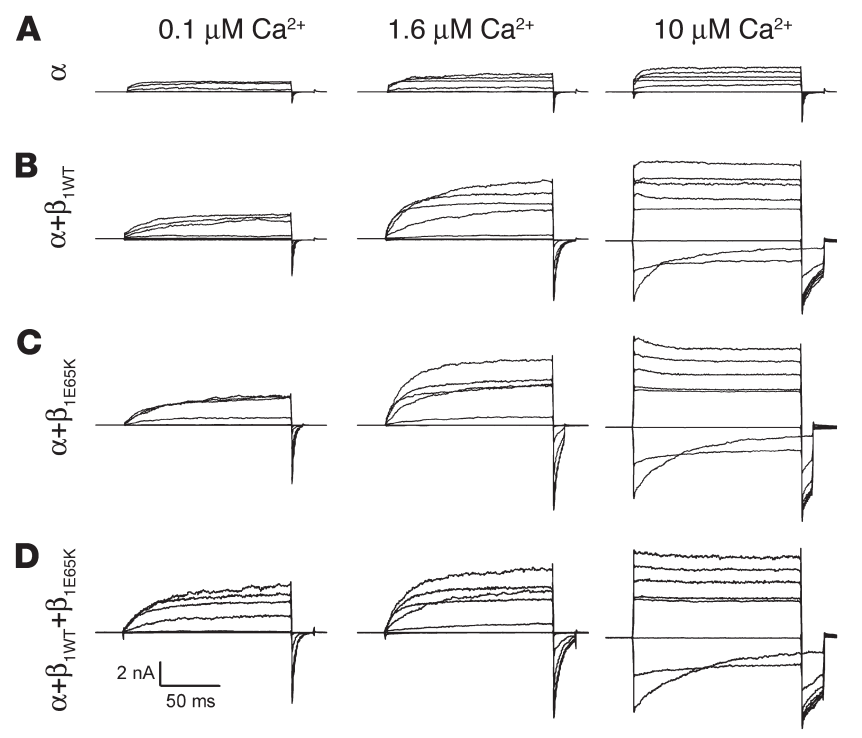

Figure 2

BK channel currents obtained from different combinations of $\alpha$ and $\beta_{1}$ BK channel subunits. Currents were recorded from excised inside-out macropatches obtained from HEK-293 cells expressing the BK $\alpha$ subunit (A), $\alpha+\beta_{1 \mathrm{WT}}(\mathbf{B}), \alpha+\beta_{1 \mathrm{E} 65 \mathrm{~K}}(\mathbf{C})$, or $\alpha+\beta_{1 \mathrm{WT}}+\beta_{1 \mathrm{EG} 6 \mathrm{KK}}$ (D). Currents were recorded at $0.1 \mu \mathrm{M}$ (left panels), $1.6 \mu \mathrm{M}$ (middle panels), and $10 \mu \mathrm{M}$ cytosolic $\mathrm{Ca}^{2+}$ (right panels). The voltage protocol was as described in Methods.

$\beta_{1 \mathrm{E} 65 \mathrm{~K}}$ alone (Figure $2 \mathrm{C}$ ) or in combination with the $\beta_{1 \mathrm{WT}}$ (Figure 2D) further enhanced the BK channel sensitivity to $\mathrm{Ca}^{2+}$, particularly with $\mathrm{Ca}^{2+}$ concentrations in the micromolar range.

The increase in the activity of BK channels is best evaluated by analysis of the conductance-voltage $(G-V)$ relationship obtained for the four experimental conditions at different $\mathrm{Ca}^{2+}$ concentrations (Figure 3). From these curves the voltage necessary to half activate the channel $\left(V_{1 / 2}\right)$ can be calculated. This is a convenient measure to evaluate the effect of $\beta_{1}$ subunits on BK channel activity, since it is directly related to the energy $(\Delta G)$ necessary to open the channel, $\Delta \mathrm{G}=z F V_{1 / 2}$, where $z$ is the apparent number of gating charges (31). The plot of the $V_{1 / 2}$ obtained for $\alpha, \alpha+\beta_{1 \mathrm{WT}}, \alpha+\beta_{1 \mathrm{E} 65 \mathrm{~K}}$, and $\alpha+\beta_{1 \mathrm{WT}}+\beta_{1 \mathrm{E} 65 \mathrm{~K}}$ currents at different cytosolic $\mathrm{Ca}^{2+}$ concentrations is shown in Figure 4. In order to facilitate the comparison between BK- $\beta_{1 \mathrm{WT}}$ and BK- $\beta_{1 \mathrm{E} 65 \mathrm{~K}}$ channel currents, Figure $4 \mathrm{~A}$ shows the $V_{1 / 2^{-}}$ versus- $\mathrm{Ca}^{2+}$ plots for $\alpha+\beta_{1 \mathrm{WT}}$ currents and $\alpha+\beta_{1 \mathrm{E} 65 \mathrm{~K}}$ currents, and Figure $4 \mathrm{~B}$ shows those for $\alpha+\beta_{1 \mathrm{WT}}$ currents and $\alpha+\beta_{1 \mathrm{WT}}+\beta_{1 \mathrm{EG5K}}$ currents. Interestingly, expression of the $\beta_{1 \mathrm{E} 65 \mathrm{~K}}$ subunit (Figure $4 \mathrm{~A}$ ) imposed a further negative shift, compared with $\beta_{1 \mathrm{WT}}$. At the highest $\mathrm{Ca}^{2+}$ concentration tested, $10 \mu \mathrm{M}$, the negative shift induced by $\beta_{1 \mathrm{E} 65 \mathrm{~K}}$ was approximately $30 \mathrm{mV}$. Coexpression of $\beta_{1 \mathrm{WT}}$ and $\beta_{1 \mathrm{E} 65 \mathrm{~K}}$ (Figure $4 \mathrm{~B}$ ) resulted in a negative shift in the $V_{1 / 2}$-versus-Ca ${ }^{2+}$ plot similar to that obtained with $\beta_{1 \mathrm{E} 65 \mathrm{~K}}$ alone. As expected, any combination of $\beta_{1}$ subunits induced a dramatic negative shift in the voltage-dependent activation of the BK channels compared with $\alpha$ currents alone (Figure 4C).

$\beta_{1 E 65 K}$ did not alter the kinetics of BK- $\beta_{1}$ channels. The presence of the $\beta_{1 \mathrm{WT}}$ subunit has also been shown to slow the activation and deactivation kinetics of BK channel currents $(11,15,30)$. Thus, we evaluated whether the kinetics of BK channel currents in the presence of $\beta_{1 \mathrm{E} 65 \mathrm{~K}}$ differed from that obtained by expression of $\beta_{1 \mathrm{WT}}$ alone. Figure $5 \mathrm{~A}$ shows representative current traces obtained from cells trans- fected with different combinations of $\alpha$ and $\beta_{1}$ subunits and normalized to the peak current, and Figure $5 \mathrm{D}$ shows representative tail currents obtained from cells expressing different combinations of $\beta_{1}$ subunits of the BK channel. Figure $5 \mathrm{~B}$ shows the plots of average activation-time constants versus voltage at $1.6 \mu \mathrm{M} \mathrm{Ca}^{2+}$, and Figure $5 \mathrm{C}$ shows the activation-time constants at $+200 \mathrm{mV}$ versus $\mathrm{Ca}^{2+}$ concentration measured in cells expressing $\alpha, \alpha+\beta_{1 \mathrm{WT}}, \alpha+\beta_{1 \mathrm{E} 65 \mathrm{~K}}$, and $\alpha+\beta_{1 \mathrm{WT}}+\beta_{1 \mathrm{E} 65 \mathrm{~K}}$. Slower kinetics were observed in $\beta_{1}$-expressing than in $\beta_{1}$-nonexpressing cells, with no difference among the $\beta_{1}$-expressing cells. Similar results were obtained when the deactivation kinetics was analyzed (Figure 5, E and F). Analysis of the time constants for the different combinations of $\beta_{1}$ subunits at different $\mathrm{Ca}^{2+}$ concentrations or voltage commands also showed no differences in the kinetics between $\beta_{1 \mathrm{WT}}$ and $\beta_{1 \mathrm{E} 65 \mathrm{~K}}$ currents (results not shown).

Allosteric modeling of the $\beta_{1 E 65 K}$ effect. Activation of BK channels is controlled by two independent stimuli: membrane voltage and intracellular $\mathrm{Ca}^{2+}$. The most recent views on the mechanisms underlying the regulation of $\mathrm{BK}$ channels by voltage and $\mathrm{Ca}^{2+}$ strongly suggest that channel opening, calcium binding, and voltage-sensor activation can be viewed as separate equilibriums that allosterically interact with each other $(26,32-37)$ (Figure 6E). Furthermore, the presence of auxiliary subunits such as the $\beta_{1}$ subunit also modifies
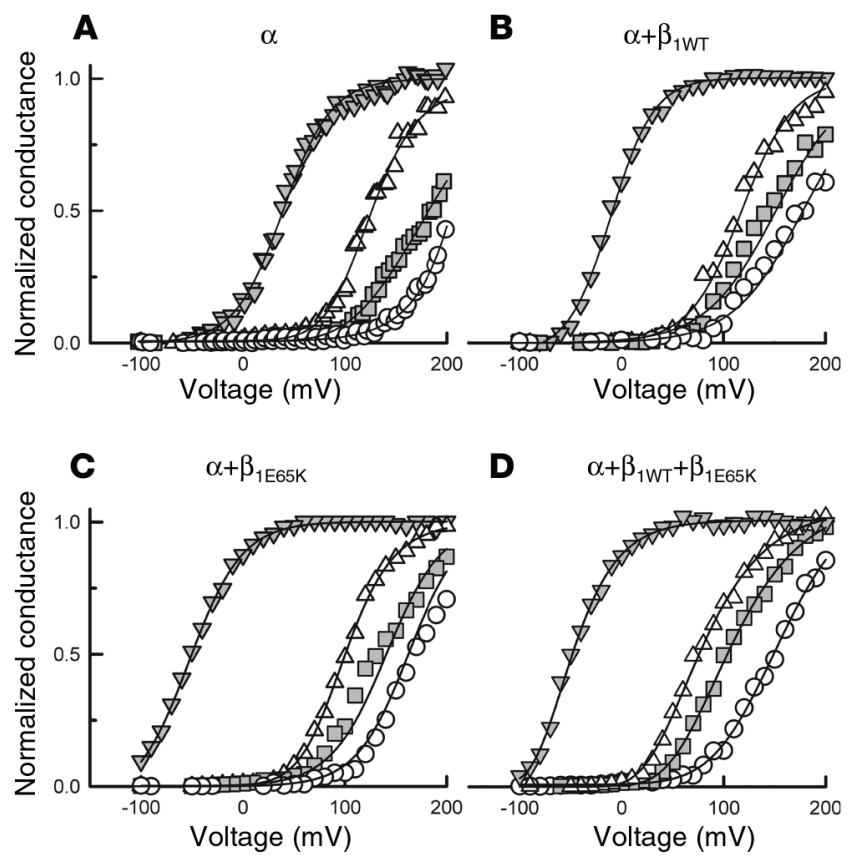

\section{Figure 3}

Plots of conductance versus voltage for BK channel currents at different $\mathrm{Ca}^{2+}$ concentrations. BK channel currents were recorded from patches excised from cells expressing $\alpha(\mathbf{A}), \alpha+\beta_{1 \mathrm{WT}}(\mathbf{B}), \alpha+\beta_{1 \mathrm{E} 65 \mathrm{~K}}$ (C), and $\alpha+\beta_{1 \mathrm{WT}}+\beta_{1 \mathrm{E} 65 \mathrm{~K}}$ (D) channel subunits. Normalized conductance was obtained for each test potential from the peak tail current at -80 $\mathrm{mV}$ measured at 0 (circles), $100 \mathrm{nM}$ (squares), $500 \mathrm{nM}$ (triangles), and $10 \mu \mathrm{M}$ (inverted triangles) $\mathrm{Ca}^{2+}$. Solid curves represent fits to the Boltzmann equation. The data at 0 and $100 \mathrm{nM} \mathrm{Ca}^{2+}$ were normalized using the value obtained at $500 \mathrm{nM}$ as maximum tail current. To obtain an average $G-V$ curve for each case, individual curves were displaced along the voltage axis by $\Delta \mathrm{V}=\left(\left\langle V_{1 / 2}\right\rangle-V_{1 / 2}\right)$ (where $\left\langle V_{1 / 2}\right\rangle$ denotes the average $V_{1 / 2}$ for each transfection group). The 300-360 resulting points were then reduced to 61 with the smoothing function of SigmaPlot (SPSS Inc., Richmond, California, USA). 

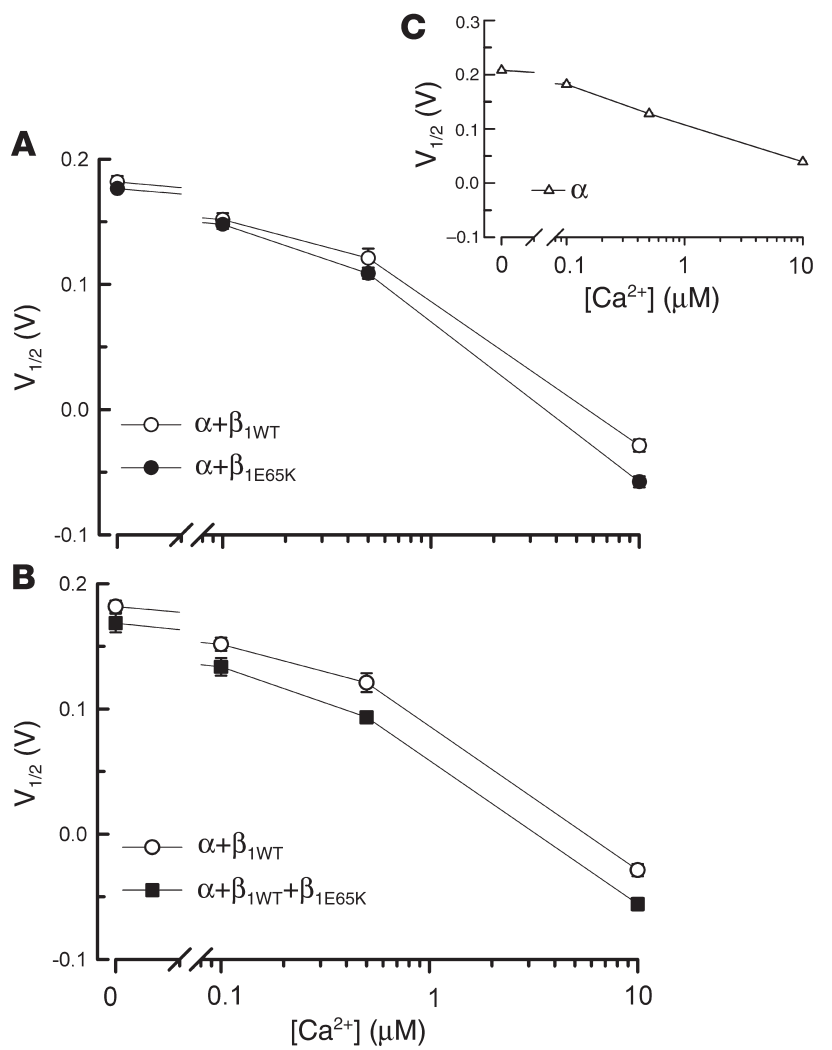

the channel gating $(13,27)$. Therefore, searching for a mechanistic explanation to the changes in the gating associated with the presence of the mutant $\beta_{1 \mathrm{E} 65 \mathrm{~K}}$ subunit, we compared our experimental data with the values provided by allosteric models $(26,27)$. The specific aim was to evaluate whether the allosteric models predict a displacement of the $G-V$ relationships without changing the parameters involved in macroscopic-current kinetics, as observed experimentally for BK- $\beta_{1 \mathrm{E} 65 \mathrm{~K}}$ channel currents when compared with BK- $\beta_{1 \mathrm{WT}}$ channel currents.

The rate-limiting step in macroscopic-current relaxation is the closed-to-open (or open-to-closed) step, since voltage-sensor movements are much faster than channel opening and closing and calcium binding is in equilibrium $(26,33-36)$. This implies that the kinetics of macroscopic-current relaxation is fully contained within the parameters that govern the open-closed transition. These parameters are the equilibrium constant $L_{0}$ and its allosteric modifiers $C$ (allosteric factor for the interaction of $\mathrm{Ca}^{2+}$ binding with

\section{Figure 5}

Effect of $\beta_{1 \mathrm{E} 65 \mathrm{~K}}$ on BK channel activation and deactivation kinetics. (A) Current traces normalized to peak current were obtained with a pulse from $0 \mathrm{mV}$ to $+200 \mathrm{mV}$ in the presence of $1.6 \mu \mathrm{M} \mathrm{Ca}^{2+}$. (B) Activation-time constants (at $1.6 \mu \mathrm{M} \mathrm{Ca}^{2+}$ ) were fitted with a single exponential function and plotted versus the pulse potential for $\alpha$ (open triangles, $n=4$ ), $\alpha+\beta_{1 \mathrm{WT}}$ (open circles, $n=13$ ), $\alpha+\beta_{1 \mathrm{E} 65 \mathrm{~K}}$ (filled circles, $n=15$ ), and $\alpha+\beta_{1 \mathrm{WT}}+\beta_{1 \mathrm{E} 65 \mathrm{~K}}$ currents (open squares, $n=6$ ). (C) Plot of activation-time constants versus $\mathrm{Ca}^{2+}$ concentrations measured with a pulse to $+200 \mathrm{mV}$. (D) Families of tail currents recorded at $1.6 \mu \mathrm{M}$ $\mathrm{Ca}^{2+}$ under the four conditions. ( $\mathbf{E}$ and $\mathbf{F}$ ) Deactivation-time constants were obtained by fitting of the tail currents with a single exponential function and plotted versus the pulse potential $(E)$ or versus the $\mathrm{Ca}^{2+}$ concentration at $-80 \mathrm{mV}(\mathbf{F})$.

\section{Figure 4}

Plot of $V_{1 / 2}$ versus $\mathrm{Ca}^{2+}$ concentration. (A) $V_{1 / 2}$ versus $\mathrm{Ca}^{2+}$ concentration obtained for $\alpha+\beta_{1 \mathrm{WT}}(n=13)$ and $\alpha+\beta_{1 \mathrm{EG} 6 \mathrm{~K}}(n=15)$. (B) $V_{1 / 2}$ versus $\mathrm{Ca}^{2+}$ concentration for $\alpha+\beta_{1 \mathrm{WT}}$ and $\alpha+\beta_{1 \mathrm{WT}}+\beta_{1 \mathrm{EE} 6 \mathrm{~K}}(n=6)$. (C) $V_{1 / 2}$ versus $\mathrm{Ca}^{2+}$ concentration for $\alpha(n=3)$. Data are shown as mean $\pm \mathrm{SEM}$. Differences in $V_{1 / 2}$ curves between different $\beta_{1}$ subunits were tested by ANOVA with repeated measurements. $P=0.002$ for $\beta_{1 \mathrm{WT}}$ Vs. $\beta_{1 \mathrm{EG} 6 \mathrm{~K}}$, and $P=0.005$ for $\beta_{1 \mathrm{WT}}$ vs. $\alpha+\beta_{1 \mathrm{WT}}+\beta_{1 \mathrm{E} 65 \mathrm{~K} \text {. }}$.

channel opening) and $D$ (allosteric factor for the interaction of voltage-sensor activation with channel opening; see Equation 1). In other words, the model predicts that any change in the parameters $J_{0}, K_{\mathrm{d}}, z_{\mathrm{L}}, z_{\mathrm{J}}$, or $E$ will affect the steady-state properties of the channel but not its macroscopic-current kinetics.

We first used the allosteric gating model to fit the $\alpha+\beta_{1 \text { WT }}$ channel data. Based on previous studies $(26,27)$, we restricted the parameters as follows: $J_{0}, K_{\mathrm{d}}$, and $L_{0}$ were restricted to values between 0.01 and 10 , between $1 \mu \mathrm{M}$ and $80 \mu \mathrm{M}$, and between $10^{-7}$ and $10^{-3}$, respectively. $z_{J}$ and $z_{L}$ were allowed to vary from 0.4 to 0.7 and from 0.2 to 0.5 , respectively. Finally, the allosteric factors $C$ and $D$ were maintained at a value greater than 1 , while $E$ was restricted to between 1 and 3 . After several runs, the best fit gave the following parameters: $J_{0}, 0.143 ; K_{\mathrm{d}}, 21.1 ; L_{0}, 1 \times 10^{-4} ; z_{\mathrm{J}}, 0.41 ; z_{\mathrm{L}}$, $0.2 ; C, 9.2 ; D, 9.7$; and $E, 3$. As can be seen in Figure 6A, the fit is in reasonable agreement with the experimental data. And although not equal, the parameters agree with those already published (27). A good fit of the $\alpha+\beta_{1 \mathrm{E} 65 \mathrm{~K}}$ data to the model, with $L_{0}, C$, and $D$ values identical to those for the wild-type condition, was also obtained (Figure 6B), and the resulting parameters were $J_{0}, 0.161$; $K_{\mathrm{d}}, 14.7 ; L_{0}, 1 \times 10^{-4} ; z_{\mathrm{J}}, 0.4 ; z_{L}, 0.2 ; C, 9.2 ; D, 9.7$; and $E$, 3 . Changes in $K_{\mathrm{d}}$ and $J_{0}$ can account for the observed displacement of the
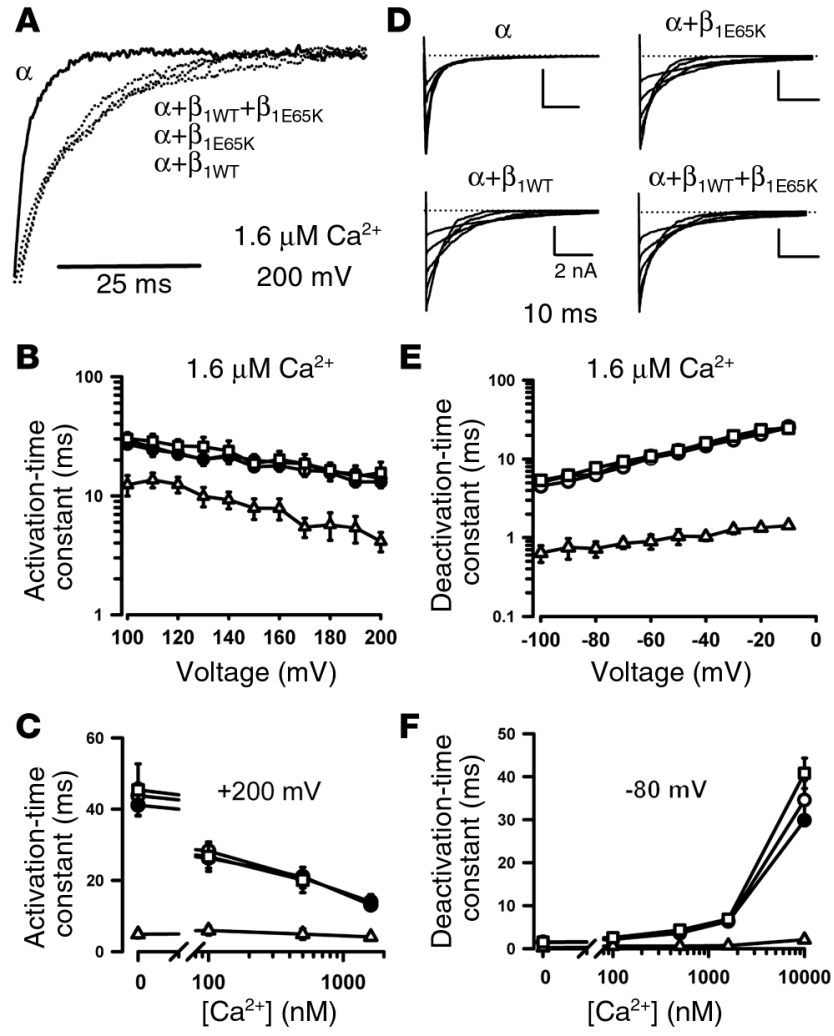

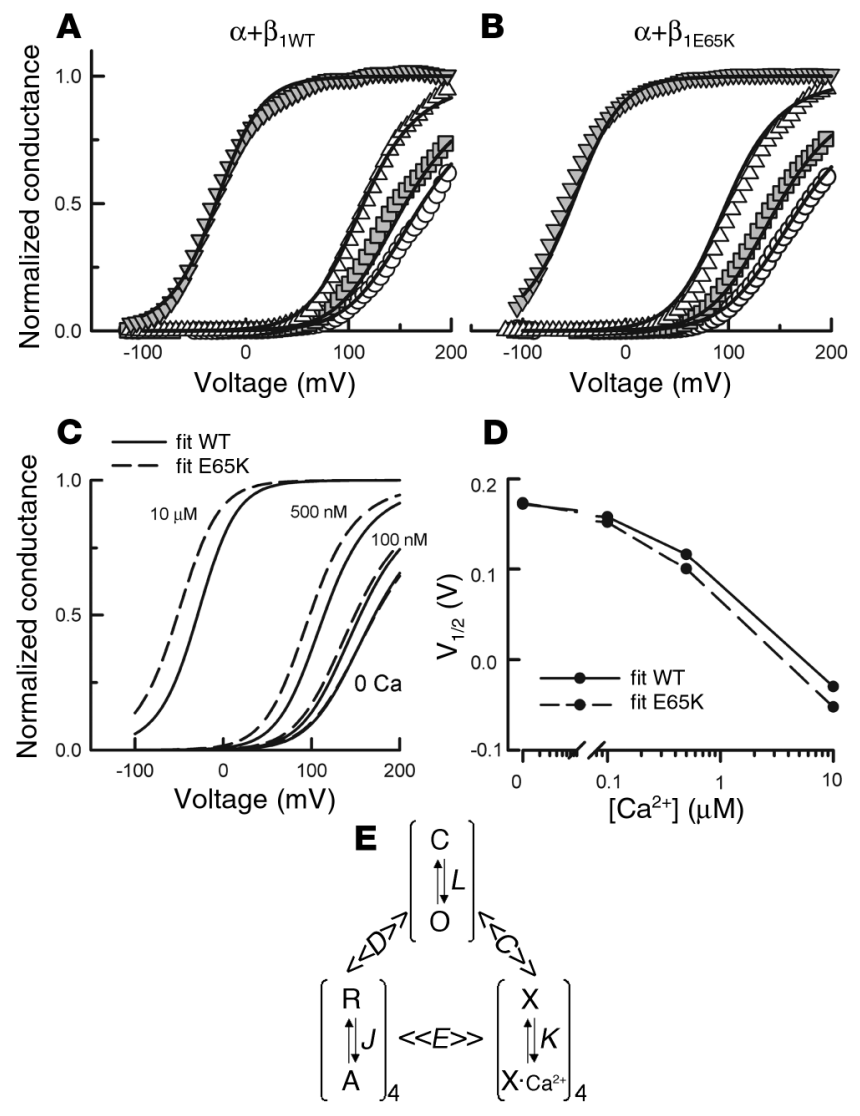

steady-state $G-V$ relationships. Figure $6 \mathrm{C}$ shows a comparison between the $G-V$ curves for the $\alpha+\beta_{1 \mathrm{WT}}$ and the $\alpha+\beta_{1 \mathrm{E} 65 \mathrm{~K}}$ predicted by the model. A comparison between the predicted $V_{1 / 2}$ for these two channels is shown in Figure 6D and is in good agreement with the experimental data shown in Figure 4B. In particular, the model predicts that the effect of the mutant $\beta_{1 \mathrm{E} 65 \mathrm{~K}}$ subunit on $V_{1 / 2}$ becomes more pronounced as the internal $\mathrm{Ca}^{2+}$ concentration is increased, and this was indeed found experimentally.

\section{Discussion}

As with most quantitative traits, differences in blood pressure result from contributions of many genes interacting with each other and with the environment. Several mutations have been identified in genes that cause rare, monogenic, forms of heritable hypertension without major repercussion at the population level. These include genes of the renin-angiotensin-aldosterone system, genes coding for adrenergic receptors, genes coding for proteins that regulate endothelial function, and genes coding for kidney ion-transport systems, among others $(3,5)$. The situation is much more complex for the $90-95 \%$ of patients suffering from essential hypertension in whom, despite enormous efforts to identify predisposing genes, no single genetic variant has emerged from linkage or association analysis as consistently related to blood pressure level in every sample and in all populations (5).

The lack of consistency in previously reported findings has prompted the experts to review the criteria for high-quality association studies and to recommend the following: the use of a large sample size, biological plausibility, functional significance, and high OR (5). Our combined functional and population-based

\section{Figure 6}

Fitting of the experimental data to an allosteric model of BK channel gating. (A and B) G-V plots for $\alpha+\beta_{1 W T}(\mathbf{A})$ and $\alpha+\beta_{1 \text { E65K }}$ (B) currents measured at 0 (circles), $100 \mathrm{nM}$ (squares), $500 \mathrm{nM}$ (triangles), and $10 \mu \mathrm{M}$ (inverted triangles) $\mathrm{Ca}^{2+}$. Solid curves represent fits to Equation 1 (see Methods) with parameters restricted as described in the text. (C) G-V plots for $\alpha+\beta_{1 \text { WT }}$ (solid line) and $\alpha+\beta_{1 \text { E65K }}$ (dashed line) channels as predicted by the model. (D) $V_{1 / 2}$-versus-Ca ${ }^{2+}$ plots obtained from the $G-V$ curves presented in $\mathbf{C}$. (E) Allosteric kinetic scheme proposed for the BK channel by Horrigan and Aldrich $(33,34)$. The C-O transition corresponds to the closed-open equilibrium where $L=L_{0} \exp \left(z_{L} \times V / k T\right)$. The R-A transition corresponds to the resting-active equilibrium of a single voltage sensor where $J=J_{0} \exp \left(z_{J} \times V / k T\right)$. The $\mathrm{X} \cdot \mathrm{Ca}^{2+}$ transition is calcium binding to a single calcium sensor, with equilibrium constant $K=\left[\mathrm{Ca}^{2+}\right] / K_{\mathrm{d}}$. These three equilibriums are related to each other by the allosteric factors $C, D$, and $E$, as shown. When there are $n$ voltage sensors active, the $\mathrm{C}-\mathrm{O}$ equilibrium constant is $L D^{n}$. Conversely, when the channel is open, the R-A equilibrium constant is $J D$. The same applies for the allosteric factors $C$ and $E$.

genetic epidemiological studies complied with the above-mentioned criteria and provide evidence for a direct involvement of the BK channel in the control of DBP in humans. A clear correlation between the genotype frequency of the $\beta_{1 \mathrm{E} 65 \mathrm{~K}}$ mutant and DBP was found. Moreover, we have shown evidence of, to our knowledge, the strongest association between a single mutation and low prevalence of moderate-to-severe diastolic hypertension. Our results are consistent with a possible protective effect of the $\mathrm{K}$ allele against the severity of diastolic hypertension and with a progressively deleterious effect of the EE genotype.

The basal vascular tone depends on calcium ions regulating the contraction of vascular smooth muscle (Figure 7B). Broad and global elevation in intracellular $\mathrm{Ca}^{2+}$ levels, mainly achieved by the activation of voltage-gated L-type calcium channels following membrane depolarization (Figure 7B, b3), induces maintained contraction (8). On the other hand, local $\mathrm{Ca}^{2+}$ transients (Figure 7B, b2), caused by the opening of a cluster of ryanodine receptor $\mathrm{Ca}^{2+}$ channels in the sarcoplasmic reticulum, only activate a group of nearby BK channels (Figure 7B, b2), without major repercussion on total intracellular $\mathrm{Ca}^{2+}$ levels. BK channel activation hyperpolarizes the cell membrane, preventing a large influx of $\mathrm{Ca}^{2+}$ via the depolarization-activated L-type calcium channels. The presence of the $\beta_{1}$ subunit increases the $\mathrm{Ca}^{2+}$ sensitivity of the $\mathrm{BK}$ channel, manifested as a negative shift in the channel's conductance-voltage relationship, making the negative-feedback mechanism more efficient and determining the basal tone (11,13-17). Recently, the role of the $\beta_{1}$ subunit in the maintaining of the basal tone has been elegantly shown with the use of $\beta_{1}$ knockout animals $(16,17)$. These models, lacking the $\beta_{1}$ subunit, represent a situation similar to that shown in Figure 7A; they face a reduction in the functional coupling of $\mathrm{Ca}^{2+}$ sparks to $\mathrm{BK}$ channel activation, and, therefore, a diminished negative-feedback mechanism.

Functional analysis of BK- $\beta_{1 \mathrm{E} 65 \mathrm{~K}}$ channel currents revealed a further negative shift in the $G-V$ relationships that becomes larger with progressive increases in intracellular $\mathrm{Ca}^{2+}$. At the highest $\mathrm{Ca}^{2+}$ concentration tested $(10 \mu \mathrm{M})$, we measured an approximately $30-\mathrm{mV}$ negative shift, compared with BK- $\beta_{1 \mathrm{wT}}$ channel currents. This consistent, negative shift in the activation of the BK- $\beta_{1 \mathrm{E} 65 \mathrm{~K}}$ channel (the $G-V$ relationships) represents an increased activity (open probability, $P_{\mathrm{O}}$ ) of the channel at equivalent voltage and $\mathrm{Ca}^{2+}$ concentrations, compared with the BK- $\beta_{1 \mathrm{WT}}$ channel. Similar 


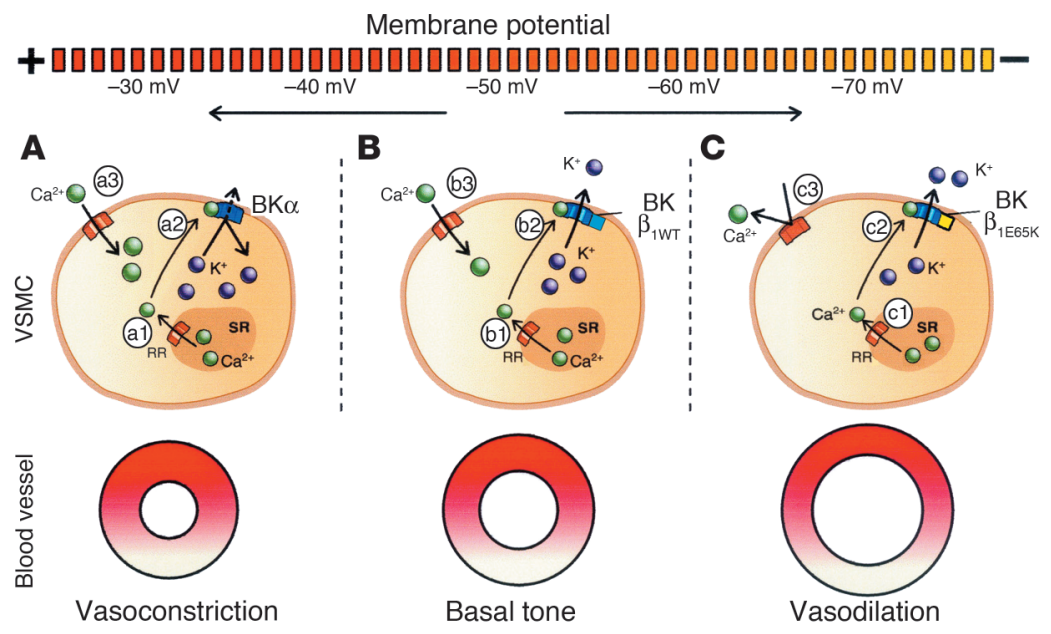

\section{Figure 7}

Proposed effect of BK- $\beta_{1 \mathrm{E} 65 \mathrm{~K}}$ channels in VSMCs. (A) Absence of the $\beta_{1}$ subunit shifts the basal tone toward a more contracted state. Ca ${ }^{2+}$ sparks (a1) caused by the opening ryanodine receptors (RR) in the sarcoplasmic reticulum (SR) activate BK channels (a2); however, because of the absence of the $\beta_{1}$ subunit, there is a decreased coupling of $\mathrm{Ca}^{2+}$ sparks to BK channel activation, resulting in low BK channel activity, membrane depolarization, and higher $\mathrm{Ca}^{2+}$ entry via depolarization-activated $\mathrm{Ca}^{2+}$ channels (a3). (B) $\mathrm{Ca}^{2+}$ sparks (b1) in the presence of the $\beta_{1}$ subunit induced a larger activation of BK- $\beta_{1}$ channels (b2), leading to an average 20-mV hyperpolarization and thus reducing the entry of Ca ${ }^{2+}$ (b3). (C) The mutant BK- $\beta_{1 \mathrm{E} 65 \mathrm{~K}}$ channels (c2) show increased activity (compared with BK- $\beta_{1 \mathrm{WT}}$ channels) at the Ca ${ }^{2+}$ concentrations produced within a single spark (c1), resulting in increased hyperpolarization and reduced activation of voltage-gated $\mathrm{Ca}^{2+}$ channels (c3), which further reduces $\mathrm{Ca}^{2+}$ entry (c3), offering a more efficient negative-feedback mechanism and, hence, vasodilation.

results, without statistical differences, were obtained when the $\beta_{1 \mathrm{E} 65 \mathrm{~K}}$ subunit was expressed alone or with the $\beta_{1 \mathrm{WT}}$ subunit, suggesting a dominant-positive effect of the mutant subunit. Such a dominant effect when the mutant allele is coexpressed with the wild-type allele has already been reported for other channels (38). Additional work, outside the scope of this study, will be necessary to clarify the mechanism by which the $\beta_{1 \mathrm{E} 65 \mathrm{~K}}$ subunit exerts its dominant-positive effect on the $\beta_{1 \mathrm{WT}}$ subunit.

In the context of vascular-tone regulation, the presence of $\beta_{1 \mathrm{E} 65 \mathrm{~K}}$ might imply a more efficient feedback mechanism, leading to a reduced $\mathrm{Ca}^{2+}$ entry via voltage-dependent $\mathrm{Ca}^{2+}$ channels (Figure $7 \mathrm{C})$. Figure 6 shows that the displacement of the $G-V$ relationships produced by the $\mathrm{E} 65 \mathrm{~K}$ mutation can be reproduced by the 70 -state allosteric model, without significant changes in the parameters involved in macroscopic-current kinetics. The 70-state model fit also allows us to make a prediction about the physiological impact of the E65K mutation. As mentioned above, smooth muscle vasoregulation mediated by the $\mathrm{BK}$ channel is mainly through spontaneous transient outward $\mathrm{K}^{+}$currents induced by ryanodine receptor-mediated $\mathrm{Ca}^{2+}$ sparks (8). Therefore, the increase in $P_{\mathrm{O}}$ upon a $\mathrm{Ca}^{2+}$ spark at the physiological resting potential is essential if a fine tuning of the smooth muscle tone is required. Recent estimates, based on the $P_{\mathrm{O}}$ increase of $\mathrm{BK}$ channels, suggest that a single $\mathrm{Ca}^{2+}$ spark raises the local calcium concentration (in microdomains just below BK channels) from $100 \mathrm{nM}$ to a value between $4 \mu \mathrm{M}$ and $30 \mu \mathrm{M}$ (39). At the physiological membrane potential of $-40 \mathrm{mV}$ and $100 \mathrm{nM} \mathrm{Ca}{ }^{2+}$, the fits predict $P_{\mathrm{O}}=0.0007$ for $\beta_{1 \mathrm{WT}}$ and 0.0009 for $\beta_{1 \mathrm{E} 65 \mathrm{~K}}$. This means that in the resting state, $\alpha+\beta_{1 \text { E65K }}$ channels are slightly more active than $\alpha+\beta_{1 \mathrm{WT}}$ channels. When the cytoplasmic $\mathrm{Ca}^{2+}$ concentration is raised to $4 \mu \mathrm{M}$ calcium (the lower limit of the estimate), the model predicts $P_{\mathrm{O}}=0.07$ for $\alpha+\beta_{1 \mathrm{WT}}$ (a 100 -fold increment) and 0.181 for $\alpha+\beta_{1 \mathrm{EG5K}}$ (a 200fold increment). This doubling of the BK channel open probabili- ty induced by the mutant $\beta_{1 \mathrm{E} 65 \mathrm{~K}}$ subunit could be the element responsible for the protective effect found in the population carrying the $\beta_{1 \mathrm{E} 65 \mathrm{~K}}$ mutation. Nevertheless, because BK channel activity in intact arteries is controlled by multiple factors, including expression levels of $\alpha+\beta_{1}$ subunits, phosphorylation balance, $\mathrm{Ca}^{2+}$ spark amplitude, and others $(18,19,40)$, definite proof of this hypothesis would only be possible with the analysis of vascular smooth muscle samples from K-carriers or animal models. Our observation that the E65K variant is associated with lower prevalence of diastolic but not of systolic hypertension might be related to the fact that systolic blood pressure mainly depends on systolic volume, whereas DBP reflects the arterial compliance and hence the vascular tone, the modulation of which is a key characteristic of the BK channel.

The E65K mutation resides on the extracellular loop of $\beta_{1}$, a domain involved in the binding of inhibitory toxins (41) and probably of activators of BK channels $(42,43)$. Although the molecular mechanism by which this mutation determines the gain of function of the BK channel remains unknown, two points regarding the interaction between $\alpha$ and $\beta_{1}$ subunits might be drawn from our study. First, the extracellular loop of $\beta_{1}$ plays a role in the increased $\mathrm{Ca}^{2+}$ sensitivity of the BK channel complex. Second, the increased $\mathrm{Ca}^{2+}$ sensitivity and the slow channel-gating properties conferred by the presence of the $\beta_{1}$ subunit can be molecularly dissected. That is, the $\beta_{1 \mathrm{E} 65 \mathrm{~K}}$ subunit augmented the $\mathrm{Ca}^{2+}$ sensitivity, compared with the $\beta_{1 \mathrm{WT}}$ subunit, without altering the channel kinetics. This behavior of the $\beta_{1 \mathrm{E} 65 \mathrm{~K}}$ subunit can be explained in terms of the allosteric model for the interaction between $\alpha$ and $\beta_{1}$ $(26,27)$. The fit of our data to this model shows that changes in the $V_{1 / 2}-\left[\mathrm{Ca}^{2+}\right]$ curve can be obtained without a change in the open-to-closed equilibrium constant, which determines the intrinsic energetics of the opening and closing of channels, and, therefore, the $\mathrm{K}^{+}$current kinetics. 
In summary, our combined functional and population-based genetic epidemiological studies provide what is, to our knowledge, the first evidence for a direct involvement of the BK channel in the control of DBP in humans. Moreover, we found a very strong association between the $\beta_{1 \mathrm{E} 65 \mathrm{~K}}$ mutant and low prevalence of moderate-to-severe diastolic hypertension, consistent with a gain of function of the BK- $\beta_{1 \mathrm{E} 65 \mathrm{~K}}$ channel and a protective effect of the $\mathrm{K}$ allele.

\section{Acknowledgments}

This work was supported by the Human Frontiers Science Organization, the Ministerio de Ciencia y Tecnología (Spain), Red HERACLES (Fondo de Investigaciones Sanitarias, Spain), Comissió Interdepartamental de Recerca i Innovació Tecnològica, Distinció de la Generalitat de Catalunya per a la Promoció de la Recerca Universitaria, and Fondo Nacional de Investigaciones Científicas y Tecnológicas (Chile). We thank L. Toro at the University of California-Los Angeles for the gift of the KCNMB1-expressing plasmid and A. Currid for proofreading the manuscript.

Received for publication October 20, 2003, and accepted in revised form January 13, 2004.

Address correspondence to: Miguel A. Valverde, Unitat de Senyalització Cel-lular, Departament de Ciències Experimentals i de la Salut, Universitat Pompeu Fabra, Calle Dr. Aiguader 80, Barcelona 08003, Spain. Phone: 34-93-542-2832; Fax: 34-93-542-2802; E-mail: miguel.valverde@upf.edu.
1. Mosterd, A., et al. 1999. Trends in the prevalence of hypertension, antihypertensive therapy, and left ventricular hypertrophy from 1950 to 1989 . N. Engl. J. Med. 340:1221-1227.

2. Warlow, C.P. 1998. Epidemiology of stroke. Lancet. 352(Suppl. 3):SIII1-SIII4

3. Lifton, R.P., Gharavi, A.G., and Geller, D.S. 2001. Molecular mechanisms of human hypertension. Cell. 104:545-556.

4. Doris, P.A. 2002. Hypertension genetics, single nucleotide polymorphisms, and the common disease: common variant hypothesis. Hypertension. 39:323-331.

5. Luft, F.C. 2002. Hypertension as a complex genetic trait. Semin. Nephrol. 22:115-126.

6. Touyz, R.M. 2000. Molecular and cellular mechanisms regulating vascular function and structure: implications in the pathogenesis of hypertension. Can. J. Cardiol. 16:1137-1146.

7. Cain, A.E., and Khalil, R.A. 2002. Pathophysiology of essential hypertension: role of the pump, the vessel, and the kidney. Semin. Nephrol. 22:3-16.

8. Jaggar, J.H., Porter, V.A., Lederer, W.J, and Nelson, M.T. 2000. Calcium sparks in smooth muscle. Am. J. Physiol. Cell Physiol. 278:C235-C256.

9. Toro, L., Wallner, M., Meera, P., and Tanaka, Y. 1998. Maxi-K(Ca), a unique member of the voltagegated K channel superfamily. News Physiol. Sci. 13:112-117.

10. Meera, P., Wallner, M., Song, M., and Toro, L. 1997. Large conductance voltage- and calcium-dependent $\mathrm{K}^{+}$channel, a distinct member of voltage-dependent ion channels with seven $\mathrm{N}$-terminal transmembrane segments (S0-S6), an extracellular $\mathrm{N}$ terminus, and an intracellular (S9-S10) C terminus. Proc. Natl. Acad. Sci. U. S. A. 94:14066-14071.

11. McManus, O.B., et al. 1995. Functional role of the beta subunit of high conductance calcium-activated potassium channels. Neuron. 14:645-650.

12. Tanaka, Y., Meera, P., Song, M., Knaus, H.G., and Toro, L. 1997. Molecular constituents of maxi KCa channels in human coronary smooth muscle: predominant alpha + beta subunit complexes. J. Physiol. 502:545-557.

13. Orio, P., Rojas, P., Ferreira, G., and Latorre, R. 2002. New disguises for an old channel: MaxiK channel beta-subunits. News Physiol. Sci. 17:156-161.

14. Knaus, H.G., et al. 1994. Primary sequence and immunological characterization of beta-subunit of high conductance $\mathrm{Ca}^{2+}$-activated $\mathrm{K}^{+}$channel from smooth muscle. J. Biol. Chem. 269:17274-17278.

15. Meera, P., Wallner, M., Jiang, Z., and Toro, L. 1996. A calcium switch for the functional coupling between alpha (hSlo) and beta subunits of maxi K channels. FEBS Lett. 385:127-128.
16. Brenner, R., et al. 2000. Vasoregulation by the beta 1 subunit of the calcium-activated potassium channel. Nature. 407:870-876.

17. Pluger, S., et al. 2000. Mice with disrupted BK channel beta1 subunit gene feature abnormal $\mathrm{Ca}^{2+}$ spark/STOC coupling and elevated blood pressure. Circ. Res. 87:E53-E60.

18. Amberg, G.C., Bonev, A.D., Rossow, C.F., Nelson, M.T., and Santana, L.F. 2003. Modulation of the molecular composition of large conductance, $\mathrm{Ca}^{2+}$ activated $\mathrm{K}^{+}$channels in vascular smooth muscle during hypertension. J. Clin. Invest. 112:717-724.

19. Amberg, G.C., and Santana, L.F. 2003. Downregulation of the BK channel $\beta_{1}$ subunit in genetic hypertension. Circ. Res. 93:965-971.

20. Gollasch, M., et al. 2002. The BK channel beta1 subunit gene is associated with human baroreflex and blood pressure regulation. J. Hypertens. 20:927-933.

21. Masia, R., et al. 1998. High prevalence of cardiovascular risk factors in Gerona, Spain, a province with low myocardial infarction incidence. REGICOR Investigators. J. Epidemiol. Community Health. 52:707-715.

22. Jiang, Z., Wallner, M., Meera, P., and Toro, L. 1999. Human and rodent MaxiK channel beta-subunit genes: cloning and characterization. Genomics. 55:57-67.

23. Ahring, P.K., Strobaek, D., Christophersen, P., Olesen, S.P., and Johansen, T.E. 1997. Stable expression of the human large-conductance $\mathrm{Ca}^{2+}$-activated $\mathrm{K}^{+}$ channel alpha- and beta-subunits in HEK293 cells. FEBS Lett. 415:67-70.

24. Boussif, O., et al. 1995. A versatile vector for gene and oligonucleotide transfer into cells in culture and in vivo: polyethylenimine. Proc. Natl. Acad. Sci. U. S. A. 92:7297-7301.

25. Hamill, O.P., Marty, A., Neher, E., Sakmann, B., and Sigworth, J. 1981. Improved patch-clamp techniques for high resolution current recording from cells and cell-free membrane patches. Pflugers Arch. 391:85-100.

26. Horrigan, F.T., and Aldrich, R.W. 2002. Coupling between voltage sensor activation, $\mathrm{Ca}^{2+}$ binding and channel opening in large conductance (BK) potassium channels. J. Gen. Physiol. 120:267-305.

27. Cox, D.H., and Aldrich, R.W. 2000. Role of the beta1 subunit in large-conductance $\mathrm{Ca}^{2+}$-activated $\mathrm{K}^{+}$ channel gating energetics. Mechanisms of enhanced $\mathrm{Ca}^{2+}$ sensitivity. J. Gen. Physiol. 116:411-432.

28. Cole, P., and McMahon, B. 1971. Attributable risk percent in case-control studies. Br. J. Prev. Soc. Med. 25:242-244.

29. Tseng-Crank, J., et al. 1996. Cloning, expression, and distribution of a $\mathrm{Ca}^{2+}$-activated $\mathrm{K}^{+}$channel beta-subunit from human brain. Proc. Natl. Acad.
Sci.U. S. A. 93:9200-9205.

30. Brenner, R., Jegla, T.J., Wickenden, A., Liu, Y., and Aldrich, R.W. 2000. Cloning and functional characterization of novel large conductance calciumactivated potassium channel beta subunits, hKCNMB3 and hKCNMB4. J. Biol. Chem. 275:6453-6461.

31. Monks, S.A., Needleman, D.J., and Miller, C. 1999. Helical structure and packing orientation of the S2 segment in the Shaker $\mathrm{K}^{+}$channel. J. Gen. Physiol. 113:415-423

32. Cui, J., Cox, D.H., and Aldrich, R.W. 1997. Intrinsic voltage dependence and $\mathrm{Ca}^{2+}$ regulation of mslo large conductance Ca-activated $\mathrm{K}^{+}$channels. J. Gen. Physiol. 109:647-673.

33. Horrigan, F.T., Cui, J., and Aldrich, R.W. 1999. Allosteric voltage gating of potassium channels. I. Mslo ionic currents in the absence of $\mathrm{Ca}^{2+}$. J. Gen. Physiol. 114:277-304.

34. Horrigan, F.T., and Aldrich, R.W. 1999. Allosteric voltage gating of potassium channels. II. Mslo channel gating charge movement in the absence of $\mathrm{Ca}^{2+}$.J. Gen. Physiol. 114:305-336.

35. Rothberg, B.S., and Magleby, K.L. 1999. Gating kinetics of single large-conductance $\mathrm{Ca}^{2+}$-activated $\mathrm{K}^{+}$channels in high $\mathrm{Ca}^{2+}$ suggest a two-tiered allosteric gating mechanism. J. Gen. Physiol. 114:93-124.

36. Rothberg, B.S., and Magleby, K.L. 2000. Voltage and $\mathrm{Ca} 2+$ activation of single large-conductance $\mathrm{Ca}^{2+}$ activated $\mathrm{K}^{+}$channels described by a two-tiered allosteric gating mechanism. J. Gen. Physiol. 116:75-99.

37. Magleby, K.L. 2003. Gating mechanism of BK (Slo1) channels: so near, yet so far. J. Gen. Physiol. 121:81-96.

38. Kullmann, D.L. 2002. The neuronal channelopathies. Brain. 125:1177-1195.

39. Perez, G.J., Bonev, A.D., and Nelson, M.T. 2001. Micromolar $\mathrm{Ca}^{2+}$ from sparks activates $\mathrm{Ca}^{2+}$-sensitive $\mathrm{K}^{+}$channels in rat cerebral artery smooth muscle. Am. J. Physiol. Cell Physiol. 281:C1769-C1775.

40. Schubert, R., and Nelson, M.T. 2001. Protein kinases: tuners of the $\mathrm{BK}_{\mathrm{Ca}}$ channel in smooth muscle. Trends Pharmacol. Sci. 22:505-512.

41. Hanner, M., et al. 1998. The beta subunit of the high conductance calcium-activated potassium channel. Identification of residues involved in charybdotoxin binding. J. Biol. Chem. 273:16289-16296.

42. Valverde, M.A., et al. 1999. Acute activation of Maxi$\mathrm{K}$ channels (hSlo) by estradiol binding to the $\beta$ subunit. Science. 285:1929-1931.

43. Dick, G.M., and Sanders, K.M. 2001. (Xeno)estrogen sensitivity of smooth muscle BK channels conferred by the regulatory beta1 subunit: a study of beta 1 knockout mice. J. Biol. Chem. 276:44835-44840. 